who required $>3$ months of pred treatment are older, with a shorter disease duration and a higher disease activity. Significant differences in the percentage change of BMD in forearm was found among the groups (Pred never/Pred $<3$ months/Pred $\geq 3$ months: $-2.99 \pm 4.21 /-1.05 \pm 3.10 /-0.65 \pm 3.45, p=0.045$ ). Post-hoc analysis revealed that the percentage reduction of forearm BMD was significantly less in the Pred $\geq 3$ months group compared to the Pred never group $(p=0.043$ ). After adjusting for age, gender, disease duration and baseline DAS-CRP, the changes in forearm BMD was still significantly different among the three groups $(p=0.015)$. No significant differences in the changes of hip and spine BMD were observed. Significant changes in forearm BMD were observed between baseline and month 12 only in the Pred never group $(0.54 \pm 0.08 / 0.53 \pm 0.0 p<0.001$,graph1).
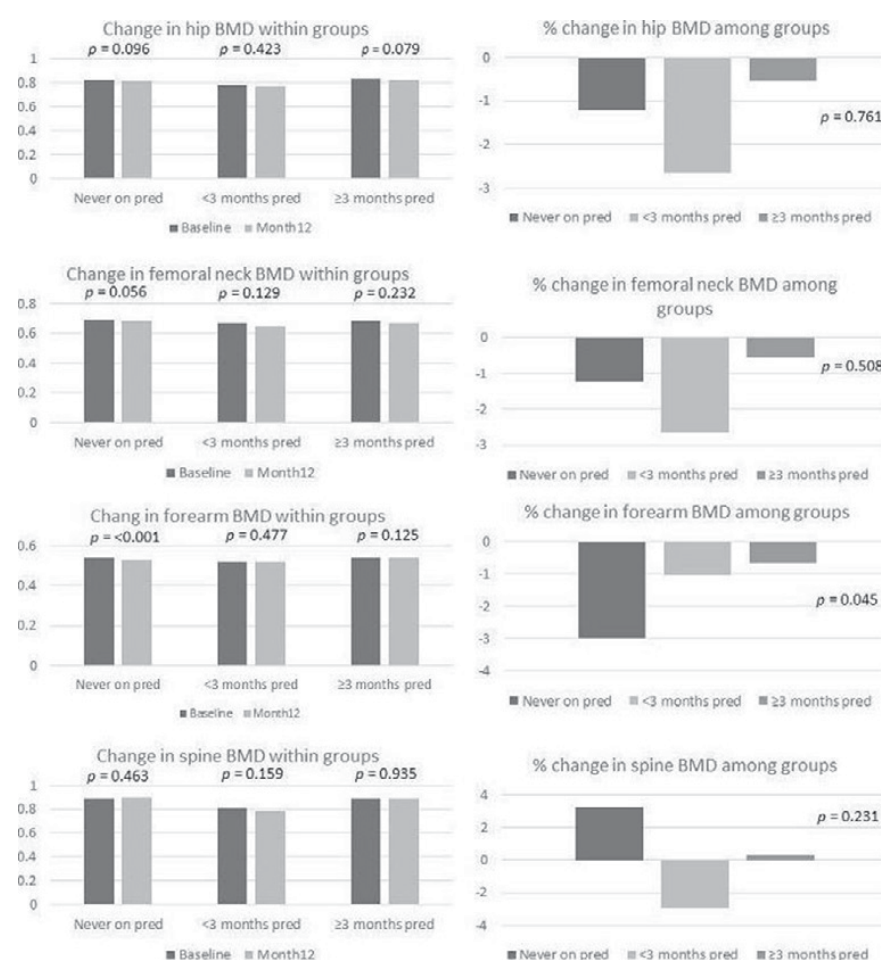

Conclusions: Small to medium dose of prednisolone might protect bone loss in forearm among early RA patients. These results need to be further validated.

References:

[1] Safety of low- to medium-dose glucocorticoid treatment in rheumatoid arthritis: myths and reality over the years. Ann N Y Acad Sci 2014.

Disclosure of Interest: None declared

DOI: 10.1136/annrheumdis-2017-eular.2842

\section{AB0222 THE SERUM ANTI-CITRULLINATED PROTEIN/PEPTIDE ANTIBODIES IN CHILDREN WITH JUVENILE IDIOPATHIC ARTHRITIS}

H. Zeng. Department of Pediatric Allergy, Immunology and Rheumatolog, Guangzhou Women and Children's Medical Center, Guangzhou, China

Objectives: To investigate the diagnostic value and prognostic usefulness of anticitrullinated protein/peptide antibodies (ACPA) in children with juvenile idiopathic arthritis (JIA).

Methods: The presence of ACPAs in the serum samples from 81 JIA patients were determined using Anti-CCP IgG, CCCP3.1 IgG and IgA ELISA. Citrullinated Protein Antibodies, Anti-MCV, and Aeskulisa RA/CP-Detect which coated various citrullinated antigen substrates: synthetic cyclic peptide, recombinant rat filaggrin, mutated human vimentin, and IgG-derived peptides. 55 children with other joint diseases and 49 healthy donors were control groups. The diagnostic performance of ACPAs was analyzed and correlations between ACPAs and radiological damage were evaluated.

Results: CPAs in $8.8-28.4 \%$ of JIA group were detected with specificity of 84.6-98.1\%. ACPAs could be seen in all subtypes of JIA, and high levels of ACPAs were particularly found in the RF positive JIA patients No healthy control had increased ACPA tested for CCP, CCP3.1, and CPA, whilst 3and 2 of healthy controls were found positive to $M C V$ and $R A / C P$, respectively. The presence of ACPAs correlated more frequently with the presence of RF $(P<0.05)$. The ACPA positivities in $18 \mathrm{JIA}$ patients with radiological damage were $27.8-55.6 \%$, which higher than that in patients without damage, and of the ACPA (CCP, CCP3.1, CPA, or MCV) positive JIA patients, $62.5 \%, 62.5 \%, 52.9 \%, 43.5 \%$ respectively had radiological damage, which significantly higher than that in JIA patients without ACPA $(P<0.05)$.

Conclusions: This study confirms the main presence of ACPAs in children with polyarticular JIA, especially those with RF positive using ELISA based methods, and ACPAs relate significantly with joint erosions in JIA.

Disclosure of Interest: None declared

DOI: 10.1136/annrheumdis-2017-eular.2058

\section{AB0223 PROGNOSTIC FACTORS FOR RADIOGRAPHIC DAMAGE IN PATIENTS WITH SERONEGATIVE RHEUMATOID ARTHRITIS}

H.Y. Lee ${ }^{1}$, E.-J. Park ${ }^{2}$, W.S. Jeong ${ }^{2}$, J. Kim ${ }^{2} .{ }^{1}$ Department of Medicine; ${ }^{2}$ Dvision of Rheumatology, Department of Medicine, Jeju National University Hospital, Jeju-si, Jeju-do, Korea, Republic Of

Background: Long since it have been suggested that seronegative rheumatoid arthritis (RA) represents a clinical entity quite distinct from that of seropositive RA. However, analytical studies dedicated to clinical outcomes regarding radiographic progression and risk factors for that are scarece ${ }^{1,2}$.

Objectives: The aim of this study is to evaluate radiographic outcome and prognostic factors for radiographic damage in patients with seronegative RA. Methods: RA pateints reportedly seronegative for both rheumatoid factor and anti-cyclic citrullinated peptide antibody who were seen at Jeju National University Hospital in South Korea between August 2003 and December 2016, and followedup at least 2 years were included. Medical records, laboratory and radiographic data was retrospectively analyzed and multivariate analysis was performed to evaluate prognostic factors for radiographic damage in patients with seronegative RA.

Results: One hundred six patients with seronegative RA were observed and 16 (15.1\%) patients demonstrated newly-developed joint damage during follow-up period. Age at diagnosis was 38.9 years and $64(60.4 \%)$ patients were female. Symptom duration at diagnosis was 1.1 years and follow-up duration was 4.4 years. Baseline characteristics including sex, symptom duration, smoking status, number of active joints, acute phase reactant, joint erosion at diagnosis were not significantly different in patients with joint damage compared to those without joint damage. Joint erosion at diagnosis and smoking status were associated with radiographic damage in seronegative RA adjusting age, symptom duration, ESR, CRP values at diagnosis, and follow-up duration, whereas it was not statistically significant (adjusted odds ratio $1.45 ; \mathrm{p}=0.061$ and $1.58 ; \mathrm{p}=0.072$ respectively) Conclusions: Our study demonstrated a rate of joint damage in patients with seronegative RA comparable to recent studies. Joint erosion at diagnosis and smoking status showed tendency to correlate with progression of radiographic damage in patients with seronegative RA. A large comparative study dedicated to this issue in seronegative RA is required.

References:

[1] Ajeganova, S. \& Huizinga, T. W. J. Nat. Rev. Rheumatol. 11, 8-9 (2015).

[2] A.G. Pratt, J.D. 652 Isaacs/Best Practice \& Research Clinical Rheumatology 28 (2014) 651e659.

Disclosure of Interest: None declared

DOI: 10.1136/annrheumdis-2017-eular.6898

\section{AB0224 SIMULATION FOR CHOICE OF BDMARDS AND TSDMARDS IN ORDER TO SUCCESS FOR THREE-YEAR SURVIVAL IN RHEUMATOID ARTHRITIS TREATMENT}

I. Yoshii. Rheumatology, Yoshii Hospital, Shimanto City, Japan

Background: In rheumatoid arthritis (RA) treatment, bDMARDs and tsDMARDs (BIO) perform tremendous disease activity control, however, its effectiveness is uncertain and unstable, because their survival ratio is not good enough to tolerate. If more right choice is done in guided by some simulation.

Objectives: This study aims more than $80 \%$ of three-year survival ratio (SR@3Y) in simulating risk of BIO by using statistical clinical data and post marketing surveillance (PMS) data with Bayes estimation.

Methods: Infection risk and survival risk were harvested from Japanese PMS data, and our clinical data. All our cases were calculated with last observation carried forward method (LOCF). If $\mathrm{BIO}$ was continued for more than three years or discontinued by attaining clinical remission, it was evaluated as success, while other cases were evaluated as failure. Patient's clinical data and general status were calculated for each case, and SR@3Y for success was statistically evaluated with Binary Logistic Analysis for success. Evaluation methods for parameters were divided according to general risk and drug specific possibility. If calculated general risk went above 0.2 , selection of $\mathrm{BIO}$ was discarded. In other case which had gone below, choice of $\mathrm{BIO}$ is done in according to point that had been cumulated by drug specific possibility in choosing what took maximum calculated expectation value.

If chosen drug have matched used BIO, it was evaluated as true, if not, it was evaluated as false, while if true case was in success, it was evaluated as true success, and if in failure, it was evaluated as true failure, while false case was in success, it was evaluated as false success, and if in failure, it was evaluated as true failure. Sensitivity in success cases and specificity in failure cases was evaluated in patients in whom BIO was administered. Statistical evaluation was done with chi-square test.

Results: 188 cases have had enough data for simulating. In these, 108 were success and 80 were failure. In success cases, simulated TNF inhibitor (TNF-i) counted 73, Tocilizumab (TCZ) counted 11, Abatacept (ABT) counted 12, and 
Tofacitinib (TOF) counted 2 while real chosen cases were TNF-i counted 65, TCZ counted 11, ABT counted 21, and TOF counted 11. Overall success ratio was $57.4 \%$. In these cases, true choice had been done in 97 cases of 108 . In failure cases, simulated TNF-i counted 44, TCZ counted 16, ABT counted 2, and TOF counted 1, while real chosen cases were TNF-i counted 49, TCZ counted 17, ABT counted 10, and TOF counted 4 . True success counted 97 , and false success counted 11, while false success counted 37 and true failure counted 54 . Then, sensitivity was $89.8 \%$ and specificity was $67.5 \%(<0.01)$.

Conclusions: Drug choice of $\mathrm{BIO}$ supported with simulation was superior to real choice. If risk management was adequately performed, SR@3Y is expected more than $85 \%$.

Disclosure of Interest: None declared

DOI: 10.1136/annrheumdis-2017-eular.6499

\section{AB0225 CHARACTERISTICS OF MHAQ FOR UPPER AND LOWER EXTREMITY FUNCTION, AND RELATIONSHIP WITH AGE AND DISEASE ACTIVITY IN RHEUMATOID ARTHRITIS PATIENT}

I. Yoshii ${ }^{1}$, T. Chijiwa ${ }^{2} .{ }^{1}$ Rheumatology, Yoshii Hospital, Shimanto City; ${ }^{2}$ Rheumatology, Kochi Memorial Hospital, Kochi, Japan

Background: Activity in daily living (ADL) is one of main target to maintain patient's quality of life in rheumatoid arthritis (RA) treatment. Modified Health Assessment Questionnaire (mHAQ) is a most popular index for ADL in routine practice. $\mathrm{mHAQ}$ is separated according to function of extremity; namely the first four categories are reflections of upper extremities (mHAQ-UE), while the latter are of lower extremities ( $\mathrm{mHAQ}-\mathrm{LE}$ ). If function of each extremity is separately disabled, it should be reflected on each part of $\mathrm{mHAQ}$.

Objectives: $\mathrm{mHAQ}$ was separately investigated in order to evaluate characteristics of each part of $\mathrm{MHAQ}$.

Methods: 964 RA patients since January 2010 had been treated. In these, patients who have been treated consecutively for more than five years at December 2016 were recruited in this study. Patient who had been operated musculoskeletal surgery was eliminated. $\mathrm{mHAQ}, \mathrm{mHAQ}-\mathrm{UE}$ and $\mathrm{mHAQ}$-LE, and 28-joint disease activity index with C-reactive protein (DAS28-CRP) were measured every time since first consult. Average value of these parameters including Sharp/van der Heijde Score (SvdHS) were calculated annually. Relationship between each of $\mathrm{mHAQ}$ and parameters for each year were evaluated used with multiple linear regression analysis.

Predominant extremity in $\mathrm{mHAQ}$ was evaluated as in what upper extremity predominant (G-UE), lower extremity predominant (G-LE), same weight (G-EV), and both of them were zero (G-Z). Changes of the evaluation from first to the last period were evaluated year by year.

Results: One hundred and two male and three hundred and thirty-three female, totally four hundred and thirty five patients were picked up. Their average value of age, SvdHS, DAS28-CRP, mHAQ, mHAQ-UE, and mHAQ-LE were 64.65, 52.1, $2.96,0.439,0.386$ and 0.491 at first consult, and $71.05,52.1,1.72,0.425,0.344$, and 0.505 at last time follow up, respectively. Both of $\mathrm{mHAQ}-\mathrm{UE}$ and $\mathrm{mHAQ}-\mathrm{LE}$ have demonstrated significant regression with both age and SvdHS throughout treatment, while not significant with DAS28-CRP, however, mHAQ-UE correlated with tenderness joint except of knee, and $\mathrm{mHAQ}$-LE have correlated with swelling of the knee joint significantly.

G-UE had counted for 85 patients, G-LE for 136 , G-EV for 49, and G-Z for 165 at first consult. Once evaluation had changed, then have continued to the last in all patients. G-UE resulted in G-UE for 83, while G-LE for 2 at last. G-LE resulted in GLE for 133, G-Z for 2, and G-EV for 1. G-EV resulted in G-LE for24, G-UE for 7, and G-EV for 18. G-Z resulted in G-Z for 137, G-LE for 19, G-UE for 4, and G-EV for 5. G-EV to G-EV demonstrated significant higher DAS28-CRP improvement from first to the last than to G-LE, and to G-UE, and G-EV to G-LE demonstrated significant higher DAS28-CRP improvement than to G-UE, although no significant difference demonstrated for mHAQ improvement among groups. G-UE to G-LE demonstrated significant higher DAS28-CRP improvement than to G-UE, as well as G-UE to G-LE demonstrated significant higher $\mathrm{mHAQ}$ improvement than to G-UE. Conclusions: From these results, it is suggested that $\mathrm{mHAQ}-\mathrm{UE}$ and $\mathrm{mHAQ}-\mathrm{LE}$ move under common influence. However, MHAQ-UE change may be reflected by upper exrtremities joint tenderness, while mHAQ-LE can move more sensitively with knee swelling. Tight disease activity control may reduce $\mathrm{mHAQ}$ both of them, however, reduces more predominantly with $\mathrm{mHAQ}-\mathrm{UE}$ than $\mathrm{mHAQ}-\mathrm{LE}$.

Disclosure of Interest: None declared

DOI: 10.1136/annrheumdis-2017-eular.2012

\section{AB0226 FUNCTIONAL DISABILITY IN RA PATIENTS TREATED WITH BIOLOGICS: HUR-BIO REAL LIFE RESULTS}

A. Sari, B. Armagan, A. Erden, L. Kilic, O. Karadag, A. Akdogan, S. Apras Bilgen, S. Kiraz, U. Kalyoncu, I. Ertenli. Rheumatology, Hacettepe University School of Medicine, Ankara, Turkey

Background: Rheumatoid arthritis (RA) is a chronic, erozive disorder which may lead to permanent joints damage. Health assessment questionnaire (HAQ) is frequently used for evaluating functional disability in RA patients.

Objectives: The aim of this study is to determine the effect of biologic treatment on functional disability in RA patients.
Methods: HUR-BIO (Hacettepe University Rheumatology Biologic Registry) is a prospective, monocentric database of biological treatments including 1229 RA patients by August 2016. 523 patients in whom HAQ assessment before biologics was avaliable, were recruited in this retrospective analysis. $\mathrm{HAQ}$ score $\geq 1.0$ was defined as severe functional disability. ${ }^{1} \mathrm{HAQ}$ assesment at last follow-up visit were evaluated. Demographic,clinical and serologic data of patients were also collected. Improvement of HAQ score 0,22 points or more was considered as clinically significant response to treatment. ${ }^{1}$

Results: Among 523 patients (80.5\% female), mean age was $52.6 \pm 12.5$ and mean disease duration was $9.4 \pm 7.3$ years. Seropositivity for RF and/or CCP was present in $67.2 \%$ of patients. At baseline visit, HAQ score was $\geq 1.0$ in 268 patients (51.2\%). Baseline and last follow-up HAQ scores were $1.07 \pm 0.62$ and $0.64 \pm 0.57$. Minimal clinically significant improvement of $\mathrm{HAQ}$ score was observed in $238 / 377$ patients $(63.1 \%)$. Clinically significant response was more frequent in patients with baseline HAQ score of $\geq 1$ (153/195 (78.4\%) vs 85/182 (46.7\%), $\mathrm{p}<0.001$ ). Table 1 represents features of patients according to baseline HAQ score. Mean follow-up time was $16.4 \pm 16.4$ months. Data of at least one visit was available for $377(72.0 \%)$ patients.

Table 1. Comparison of demographic an clinical data of patients according to baseline HAQ score

\begin{tabular}{lccc}
\hline & $\mathrm{HAQ} \geq 1(\mathrm{n}=268)$ & $\mathrm{HAQ}<1(\mathrm{n}=255)$ & $\mathrm{p}$ \\
\hline Age, mean $\pm \mathrm{SD}$ & $55.1 \pm 12.3$ & $50.0 \pm 12.3$ & $<0.001$ \\
Disease duration (years), mean $\pm \mathrm{SD}$ & $10.2 \pm 8.0$ & $8.6 \pm 6.3$ & 0.01 \\
BMI, mean $\pm \mathrm{SD}$ & $31.3 \pm 6.4$ & $28.9 \pm 5.9$ & $<0.001$ \\
High school or collage graduate $\mathrm{n}(\%)$ & $68(25.3)$ & $110(43.1)$ & $<0.001$ \\
Smoking (current or previous), $\mathrm{n}(\%)$ & $95(35.4)$ & $123(4 ., 4)$ & $<0.001$ \\
Hypertension, $\mathrm{n}(\%)$ & $101(37.7)$ & $59(23.1)$ & $<0.001$ \\
Patient global assesment VAS, mean $\pm \mathrm{SD}$ & $6.8 \pm 1.4$ & $5.7 \pm 1.8$ & $<0.001$ \\
Fatigue VAS, mean \pm SD & $6.7 \pm 2.0$ & $5.0 \pm 2.8$ & $<0.001$ \\
Pain VAS, mean \pm SD & $7.1 \pm 1.6$ & $6.1 \pm 2.1$ & $<0.001$ \\
Biologic switch, $\mathrm{n}(\%)$ & $63(23.5)$ & $41(16.1)$ & 0.033 \\
\hline
\end{tabular}

Conclusions: Functional disabilty was observed approximately half of patients. Clinically significant improvement was more frequent among patients with higher baseline HAQ scores particularly. Biologic treatment seems to be provide significant functional improvement. However significant functional disability persists in one fourth of patients.

\section{References:}

[1] Lillegraven S., Kvien T.K. Measuring disability and quality of life in established rheumatoid arthritis. Best Pract Res Clin Rheumatol. 2007;21:827-840.

Disclosure of Interest: None declared

DOI: 10.1136/annrheumdis-2017-eular.4098

\section{AB0227 IMPACT OF EARLY ARTHRITIS CLINIC ON THE RATE OF TREATMENT WITH BIOLOGICS IN RHEUMATOID ARTHRITIS: INTERRUPTED TIME SERIES ANALYSIS}

I. Farina, E. Galuppi, G. Ciancio, C. De Giorgio, C.A. Scire', M. Govoni. Department of Medical Sciences, UOL Rheumatology, Section of Haematology and Rheumatology, University of Ferrara and Azienda Ospedaliero-Universitaria Sant'Anna, Ferrara, Italy

Background: Early diagnosis is one of the mainstay of rheumatoid arthritis (RA) management. Early diagnosis reflects on early treatment, better response with reduction of long-term detrimental disease outcomes. Early arthritis clinics (EAC) are the healthcare services devoted to facilitate early diagnosis and optimize treatment of early onset inflammatory arthritis. Despite the a priori beneficial potential of EAC, no strong experimental data support EAC efficacy. Interrupted time series analysis is one of the "next best" approaches for dealing with interventions when randomisation is not possible or clinical trial data are not available.

Objectives: To evaluate the impact of the implementation of an EAC in terms of probability of starting second-line biologic DMARDs, using a quasi-experimental approach.

Methods: RA patients fulfilling 1987 ACR criteria who attended the outpatient rheumatology clinic (RC) between 2002 and 2008 and the Early Arthritis Clinic between 2009 and 2014 were retrospectively analyzed. The EAC was developed as a healthcare service integrating primary care with tertiary rheumatology care to provide early referral of suspected inflammatory arthritis and tight monitoring and standardized therapeutic approach according to "treat to target" (T2T) strategy and EULAR guidelines to early RA. The two sub-cohorts were compared in terms of: 1) lag time from symptoms onset to RA treatment with DMARDs; and 2) risk of treatment with bDMARDs at 24 months. Interrupted time analysis was performed to compare lag time from onset to treatment and log-transformed 24-month risk of biologics periods before the implementation of the EAC with subsequent periods. Results: A total of 353 RA patients were included: 208 (mean \pm SD age 58.7 \pm 12.6 years, $164 \mathrm{~F}$, baseline DAS28 $4.76 \pm 1-23$ ) followed in RC and 145 (mean \pm SD age $58.8 \pm 14.9$ years, $106 \mathrm{~F}$, DAS28 $5.09 \pm 1.31$ ) in EAC. Lag time from symptoms onset to treatment resulted significantly lower (median [IQR] months 4 [2-7] vs 12 [5-24]; $p<0.0001$ ) in patients managed in EAC compared with RC. Within 24 months a biologic therapy was started in $62 / 208(29.8 \%)$ of patients followed in $R C$, and $21 / 145(14.5 \%)$ in EAC group ( $p=0.001)$, along with an increased remission rate at 24 months ( $43 \%$ vs $62 \%, p<0.001)$. Analyzing the time series "interrupted" by the implementation of the EAC, comparing before and after 